\title{
Implementation of Subgame-Perfect Cooperative Agreement in an Extensive-Form Game*
}

\author{
Denis Kuzyutin $^{1,2}$, Yulia Skorodumova ${ }^{1}$ and Nadezhda Smirnova ${ }^{2}$ \\ 1 St. Petersburg State University, \\ 7/9 Universitetskaya nab., Saint Petersburg 199034, Russia, \\ E-mail: d.kuzyutin@spbu.ru \\ 2 HSE University, \\ St. Petersburg School of Mathematics, Physics and Computer Science \\ Soyuza Pechatnikov ul.16, St. Petersburg, 190008, Russia \\ E-mail: nvsmirnova@hse.ru
}

\begin{abstract}
A novel approach to sustainable cooperation called subgameperfect core (S-P Core) was introduced by P. Chander and M. Wooders in 2020 for $n$-person extensive-form games with terminal payoffs. This solution concept incorporates both subgame perfection and cooperation incentives and implies certain distribution of the total players' payoff at the terminal node of the cooperative history. We use in the paper an extension of the S-P Core to the class of extensive games with payoffs defined at all nodes of the game tree that is based on designing an appropriate payoff distribution procedure $\beta$ and its implementation when a game unfolds along the cooperative history. The difference is that in accordance with this so-called $\beta$-subgameperfect core the players can redistribute total current payoff at each node in the cooperative path. Moreover, a payoff distribution procedure from the $\beta$-S-P Core satisfies a number of good properties such as subgame efficiency, non-negativity and strict balance condition.

In the paper, we examine different properties of the $\beta$-S-P Core, introduce several refinements of this cooperative solution and provide examples of its implementation in extensive-form games. Finally, we consider an application of the $\beta$-S-P Core to the symmetric discrete-time alternating-move model of fishery management.
\end{abstract}

Keywords: extensive game, sustainable cooperation, subgame-perfect equilibrium, core, payoff distribution procedure, renewable resource extraction.

\section{Introduction}

A new solution concept for $n$-person games in extensive form with terminal payoffs that takes into account both cooperation incentives and subgame perfection notion was introduced in (Chander and Wooders, 2020). This solution concept called subgame-perfect core (S-P Core) is based on two specific properties of an extensive-form game and two main assumptions:

- A set of players in the subgame could be smaller than the set of all players in the original extensive-form game. It is worth noting that a specific approach to the subgame definition that takes into account only the "active" players (i.e. the players which have at least one decision node in the subgame), called "A-subgame concept" was elaborated in (Kuzyutin and Romanenko, 1998; Petrosyan and Kuzyutin, 2000; Kuzyutin et al., 2019a).

${ }^{*}$ Funding: The reported study was funded by RFBR, project number 21-011-44058.

https://doi.org/10.21638/11701/spbu31.2021.19 
- "Bargaining power" of some coalition (guaranteed or achievable payoff of this coalition) may vary, i.e., be higher or lower as a game unfolds along the history generated by a strategy profile.

- If a coalition $S$ deviates from the cooperative scenario in a subgame (all players in $S$ should be active in this subgame) the remaining players do not form any coalitions, and achievable payoff of coalition $S$ is equal to the (highest) subgameperfect equilibrium payoff of $S$ in the "induced" game between $S$ and remaining separate players. Such assumption was used in (Chander and Tulkens, 1997; Chander, 2007) to introduce so-called $\gamma$-characteristic function for cooperative games.

- The payoffs are transferable and, hence, the players can promise to redistribute maximal total payoff of the grand coalition between the players (in the corresponding terminal node) to sustain a cooperative scenario.

A natural extension of the subgame-perfect core concept to more broad class of extensive games (namely, when the player's payoffs are determined at all the nodes) was proposed in (Kuzyutin and Smirnova, 2021). This cooperative solution (called $\beta$-S-P Core) implies designing an appropriate payoff distribution procedure (PDP) (see, e.g., Petrosyan and Danilov, 1979; Petrosyan and Kuzyutin, 2000; Yeung and Petrosyan, 2012; Haurie et al., 2012; Kuzyutin et al., 2018) that determines a rule of the current total payoffs redistribution at each node along the cooperative path and satisfies a number of good properties.

In the paper, we are mainly focused on the following issues:

- If we compare two closely related cooperative solutions - subgame-perfect core (Chander and Wooders, 2020) and $\beta$-S-P Core (Kuzyutin and Smirnova, 2021) - which of them provides more powerful tool to sustain the cooperative agreement?

- Is there a relationship between strong Nash equilibria (SNE) and $\beta$-S-P Core for the class of extensive-form games under consideration?

- If $\beta$-S-P Core consists of multiple payoff distribution procedures (in general, this is exactly the case due to Prop. 3), what approaches for the refinement/contraction of the $\beta$-S-P Core could be proposed?

We provide answers to these questions below. Besides, we demonstrate the $\beta$ S-P Core implementation for sustaining a cooperative scenario in the symmetric version of the discrete-time fishery-management model (Levhari and Mirman, 1980; Haurie et al., 2012; Kuzyutin and Smirnova, 2021).

The remainder of the paper is organized as follows: the class of extensive-form games with perfect information is presented in Section 2. In Section 3, we provide the S-P Core definition due to (Chander and Wooders, 2020) as well as an example of extensive game with terminal payoffs and empty S-P Core. Then, in Section 4, we treat the same game as the game with payoffs defined at each node of the game tree and find out that $\beta$-S-P Core is non-empty and consists of multiple PDP. In Section 5 , we reveal the relationship between SNE and $\beta$-S-P Core. Several approaches to the $\beta$-S-P Core refinement are proposed in Section 6 . We consider $\beta$-S-P Core implementation in the symmetric two-stage fishery-management model in Section 7 and briefly conclude in Section 8 . 


\section{Extensive-form game with perfect information}

We consider a finite multistage game in extensive form following (Kuhn, 1953; Petrosyan and Kuzyutin, 2000; Kuzyutin and Romanenko, 1998; Kuzyutin and Smirnova, 2020). First we need to define the basic notations that will be used in the sequel:

$-N=\{1, \ldots, n\}$ is the finite set of all players.

- $K$ is the game tree with the root (origin) $x_{0}$ and the set of all nodes $P$.

$-S(x)$ is the set of all direct successors of the node $x$, and $S^{-1}(y)$ is the unique predecessor (parent) of the node $y \neq x_{0}$ such that $y \in S\left(S^{-1}(y)\right)$.

- $P_{i}$ is the set of all decision nodes of the $i$ th player (at these nodes the player $i$ chooses the following node), $P_{i} \cap P_{j}=\emptyset$, for all $i, j \in N, i \neq j$.

$-P_{n+1}=\left\{z^{j}\right\}_{j=1}^{m}$ denotes the set of all terminal nodes (final positions), $S\left(z^{j}\right)=\emptyset$ $\forall z^{j} \in P_{n+1}$. Note that $\bigcup_{i=1}^{n+1} P_{i}=P$.

$-\omega=\left(x_{0}, \ldots, x_{t-1}, x_{t}, \ldots, x_{T}\right)$ is the history (or the path) in the game tree, $x_{t-1}=S^{-1}\left(x_{t}\right), 1 \leq t \leq T, x_{T}=z^{j} \in P_{n+1}$; where "time index" $t$ in $x_{t}$ denotes the ordinal number of this node in the path $\omega$.

$-h_{i}(x)$ is the payoff of the $i$ th player at node $x \in P$. We suppose that the payoffs are non-negative, that is, $h_{i}(x) \geqslant 0$ for all $i \in N$, and $x \in P$.

Let $G^{P}(n)$ denote the class of all finite $n$-person extensive-form games with perfect information (see, e.g., Kuhn, 1953; Petrosyan and Kuzyutin, 2000; Haurie et al., 2012), while $\Gamma^{x_{0}} \in G^{P}(n)$ denotes a game with origin $x_{0}$.

Since all the solutions we are interested in throughout the paper are attainable when the players restrict themselves to the class of pure strategies we will focus on this class of strategies. The pure strategy $u_{i}(\cdot)$ of the $i$ th player for each node $x \in P_{i}$ specifies the next node $u_{i}(x) \in S(x)$ which the player $i$ has to select at $x$. Let $U_{i}$ denote the set of all pure strategies of the $i$-th player, $U=\prod_{i \in N} U_{i}$. Each strategy profile $u=\left(u_{1}, \ldots, u_{n}\right) \in U$ generates a path $\omega(u)=\left(x_{0}, \ldots, x_{t}, x_{t+1}, \ldots, x_{T}\right)=$ $\left(x_{0}, x_{1}(u), \ldots, x_{t}(u), x_{t+1}(u), \ldots, x_{T}(u)\right)$, where $x_{t+1}=u_{j}\left(x_{t}\right) \in S\left(x_{t}\right)$ if $x_{t} \in P_{j}$, $0 \leq t \leq T-1, x_{T} \in P_{n+1}$, and, hence, a vector of the players' payoffs. Lastly, let

$$
H_{i}(u)=\tilde{h}_{i}(\omega(u))=\sum_{\tau=0}^{T} h_{i}\left(x_{\tau}(u)\right)
$$

denote the value of the $i$ th player's payoff function given strategy profile $u$.

According to (Kuhn, 1953; Petrosyan and Kuzyutin, 2000; Haurie et al., 2012) each decision node $x_{t} \in P \backslash P_{n+1}$ generates a subgame $\Gamma^{x_{t}}$ with the subgame tree $K^{x_{t}}$. Let $P_{i}^{x_{t}}, i \in N$, denote the restriction of $P_{i}$ on the subgame tree $K^{x_{t}}$, and $u_{i}^{x_{t}}, i \in N$, denote the restriction of the $i$ th player's pure strategy $u_{i}(\cdot)$ in $\Gamma^{x_{0}}$ on $P_{i}^{x_{t}}$. The (subgame) strategy profile $u^{x_{t}}=\left(u_{1}^{x_{t}}, \ldots, u_{n}^{x_{t}}\right)$ determines the path $\omega^{x_{t}}\left(u^{x_{t}}\right)=\left(x_{t}, x_{t+1}, \ldots, x_{T}\right)=\left(x_{t}, x_{t+1}\left(u^{x_{t}}\right), \ldots, x_{T}\left(u^{x_{t}}\right)\right)$ in the subgame and, correspondly, a vector of the player's payoffs in the subgame $\Gamma^{x_{t}}$ :

$$
H_{i}^{x_{t}}\left(u^{x_{t}}\right)=\tilde{h}_{i}^{x_{t}}\left(\omega^{x_{t}}\left(u^{x_{t}}\right)\right)=\sum_{\tau=t}^{T} h_{i}\left(x_{\tau}\left(u^{x_{t}}\right)\right) .
$$

Note that (1) essentially differs from the subgame payoff definition that is accepted for the games with terminal payoffs in extensive form (see, e.g., Kuhn, 1953; Petrosyan and Kuzyutin, 2000). 
Definition 1. (Nash, 1950) A strategy profile $u=\left(u_{1}, u_{2}, \ldots, u_{n}\right)$ is a Nash Equilibrium (NE) in $\Gamma^{x_{0}} \in G^{P}(n)$, if $H_{i}\left(v_{i}, u_{-i}\right) \leqslant H_{i}\left(u_{i}, u_{-i}\right), \forall v_{i} \in U_{i}, \forall i \in N$.

Let $N E\left(\Gamma^{x_{0}}\right)$ denote the set of all pure strategy Nash equilibria in $\Gamma^{x_{0}}$.

Definition 2. (Selten, 1975) A strategy profile $u$ is a subgame perfect (Nash) equilibrium (SPE) in $\Gamma^{x_{0}} \in G^{P}(n)$, if $\forall x \in P \backslash P_{n+1}$ it holds that $u^{x} \in N E\left(\Gamma^{x}\right)$, i.e. the restriction of $u$ on each subgame $\Gamma^{x}$ forms a NE in this subgame.

\section{S-P Core in an extensive game with terminal payoffs}

The novel and promising concept of consistent cooperative behavior called subgame-perfect core was introduced in (Chander and Wooders, 2020) for extensive form games with perfect information (the authors focus on the special type of extensiveform games when the players payoffs are defined and, hence, could be redistributed only in terminal nodes). For the sake of completeness we provide a subgame-perfect core definition below following (Chander and Wooders, 2020). Namely, the player $i \in N$ is called active in the subgame $\Gamma^{x}$ if this player has at least one decision node in this subgame, i.e. $P_{i} \cap K^{x} \neq \emptyset$. Similarly, we shall refer to coalition $S \subset N$ as active coalition in $\Gamma^{x}$ (or simply "active at $x^{\prime \prime}$ ) if all the players from $S$ are active in $\Gamma^{x}$.

Given coalition $S \subset N$ let $\Gamma^{x_{0}, S}$ denote the induced game, which differs from $\Gamma^{x_{0}}$ only in that coalition $S$ becomes a new player with $h_{S}(x)=\sum_{i \in S} h_{i}(x), x \in P^{n+1}$.

The induced subgame $\Gamma^{x, S}$ is defined in the same manner given that coalition $S$ is active in $\Gamma^{x}$.

Denote by $\gamma(S ; x), x \in P \backslash P_{n+1}, S \subset N$, the highest possible subgame-perfect equilibrium payoff of $S$ in the induced game $\Gamma^{x, S}$. In an extensive-form game with terminal payoffs a payoff vector $\left(p_{1}, \ldots, p_{n}\right)$ is feasible if $\sum_{i \in N} p_{i}=\sum_{i \in N} h_{i}(z)=$ $h_{N}(z)$ for some terminal node $z \in P_{n+1}$. Note that for any terminal node $z \in$ $P_{n+1}$ there exists a unique history $\omega=\left(x_{0}, \ldots, z\right)$ leading to this node. Following (Chander and Wooders, 2020) we will refer to a history leading to $z \in P_{n+1}$ as a history leading to the feasible payoffs vector $\left(p_{1}, \ldots, p_{n}\right)$, given that $\sum_{i \in N} p_{i}=h_{N}(z)$.

Definition 3. (Chander and Wooders, 2020) A feasible payoff vector $\left(p_{1}, \ldots, p_{n}\right)$ belongs to the subgame-perfect core (S-P Core) of a game $\Gamma \in G^{P}(n)$ in extensive form with terminal payoffs, if for all decision nodes $x$ along any history $\omega=\left(x_{0}, \ldots, z\right)$ leading to $\left(p_{1}, \ldots, p_{n}\right), \sum_{i \in N} p_{i}=h_{N}(z)$ and for all coalitions $S \subset N$ which are active at $x$ the following inequality holds

$$
\gamma(S ; x) \leqslant \sum_{i \in S} p_{i}
$$

Remark 1. Definition 3 implies (see (Chander and Wooders, 2020) for details) that any feasible payoff vector $\left(p_{1}, \ldots, p_{n}\right)$ from the subgame-perfect core is efficient, i.e.

$$
\sum_{i \in N} p_{i}=\max _{z \in P_{n+1}} \sum_{i \in N} h_{i}(z)=h_{N}\left(z^{*}\right) .
$$

Moreover, (2) means that no coalition $S$ which is active in any subgame $\Gamma^{x}$ along the histories leading to all the terminal nodes $z^{*}$ meeting (3) can get a SPE 
payoff $\gamma(S ; x)$ that is higher than its total payoff in a subgame-perfect core vector $\left(p_{1}, \ldots, p_{n}\right)$.

The total payoff (3) could be interpreted as a (maximal) cooperative payoff of the grand coalition $N$ while any history $\left(x_{0}, \ldots, z^{*}\right)$ is a cooperative history. Then, S-P Core payoff vector $\left(p_{1}, \ldots, p_{n}\right)$ is an imputation (distribution of the cooperative payoff among the players) that is consistent in a sense of (2) in any subgame $\Gamma^{x}$ along any cooperative history. Hence, if the S-P Core of an extensive-form game $\Gamma \in G^{P}(n)$ with terminal payoffs is non-empty, the players can ensure the sustainability of the cooperative scenario via redistribution of the total payoff $h_{N}\left(z^{*}\right)$ at all terminal nodes $z^{*}$ meeting (3) according to the subgame-perfect core vector $\left(p_{1}, \ldots, p_{n}\right)$.

Let us use the following example to demonstrate the S-P Core features.

Example 1. (A 2-player extensive-form game with terminal payoffs: S-P Core).

Let $n=2, P_{n+1}=\left\{z_{1}, \ldots, z_{8}\right\}, P_{1}=\left\{x_{0}, x_{3}, x_{4}, x_{5}, x_{6}\right\}, P_{2}=\left\{x_{1}, x_{2}\right\}$. Firstly, consider this extensive-form game as a game with terminal payoffs, i.e. the players payoffs $h_{i}(z)$ are defined only in terminal nodes $z \in P_{n+1}$. This payoffs $\left(h_{1}(z), h_{2}(z)\right)^{T}$ are written in rectangles below each terminal node, while we assume zero players' payoffs for all decision nodes $x_{0}, \ldots, x_{6}$.

The cooperative history $\bar{\omega}=\left(x_{0}, x_{2}, x_{6}, z_{7}=z^{*}\right)$, which is marked in bold in fig.1, corresponds to the maximal total (cooperative) payoff 24. This game possesses a unique subgame-perfect equilibrium (the corresponding choices of the players at all decision nodes are marked as dotted lines) that determines a path $\omega=\left(x_{0}, x_{1}, x_{3}, z_{1}\right)$ and payoff vector $(14,8)$.

Obviously, $p_{1}+p_{2}=24$ according to (3), and the S-P Core is empty since

$$
\gamma\left(\{1\} ; x_{0}\right)=14 \leqslant p_{1}, \quad \gamma\left(\{2\} ; x_{2}\right)=12 \leqslant p_{2} .
$$

Hence, the players can not ensure the sustainability of the cooperative scenario by means of only redistributing the cooperative payoff at terminal node $z_{7}=z^{*}$.

However, we will use this example to motivate the way of the S-P Core concept extension to more broad class of extensive-form games. Namely, if the players payoffs are determined at all the nodes, and the players can redistribute the total current payoff at every node in the cooperative path, a natural modification of the S-P Core definition allows to ensure a non-emptiness of the (modified or extended) S-P Core for the game in Ex. 1 as well as to provide the algorithm of its implementation via an appropriate payoff distribution procedure (see, for instance (Petrosyan and Danilov, 1979; Petrosyan and Kuzyutin, 2000; Haurie et al., 2012)).

\section{Payoff distribution procedure approach and $\beta$-subgame-perfect core}

Hereinafter we consider the general case of an extensive-form game $\Gamma^{x_{0}} \in G^{P}(n)$ when the players payoffs $h_{i}(x)$ are determined at all nodes $x \in P$. Let $\bar{\omega}=\bar{\omega}(\bar{u})=$ $\left(x_{0}=\bar{x}_{0}, \ldots, \bar{x}_{t}, \ldots, \bar{x}_{T}\right)$ denote a cooperative (history), i.e.

$$
\max _{u \in U} \sum_{i \in N} H_{i}(u)=\sum_{i \in N} H_{i}(\bar{u})=\sum_{i \in N} \sum_{\tau=0}^{T} h_{i}\left(\bar{x}_{\tau}\right)=\sum_{i \in N} \widetilde{h}_{i}(\bar{\omega}) .
$$

Suppose that there exists a unique cooperative path in $\Gamma^{x_{0}} \in G^{P}(n)$. Otherwise, we assume that the players use a specific approach (for instance, the PRB 


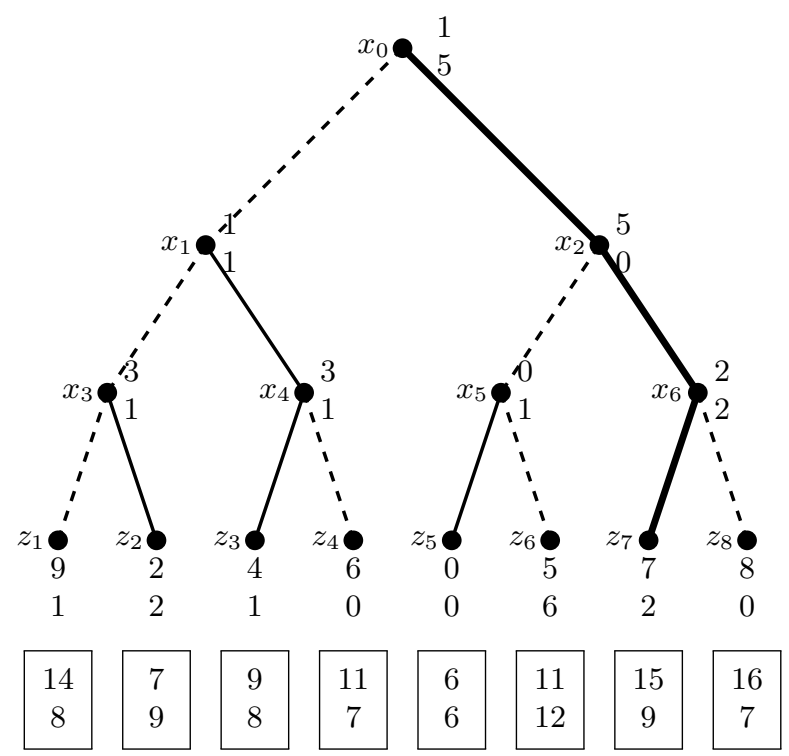

Fig. 1. 2-person extensive-form game

algorithm proposed in (Kuzyutin and Smirnova, 2020)) to select a unique cooperative path from all the histories $\bar{\omega}$ meeting (4). It is worth noting that PRB algorithm was proved to satisfy time consistency property (see, e.g., Petrosyan, 1977; Petrosyan and Danilov, 1979; Petrosyan and Kuzyutin, 2000; Kuzyutin and Smirnova, 2020).

A vector $\left(p_{1}^{\bar{x}_{t}}, \ldots, p_{n}^{\bar{x}_{t}}\right)$ such that

$$
\sum_{i \in N} p_{i}^{\bar{x}_{t}}=\sum_{i \in N} \sum_{\tau=t}^{T} h_{i}\left(\bar{x}_{\tau}\right)=\sum_{i \in N} \widetilde{h}_{i}\left(\bar{\omega}^{\bar{x}_{t}}\right)
$$

determines a possible distribution of the total cooperative (subgame) payoff among the players and could be considered as a cooperative solution for the subgame $\Gamma^{\bar{x}_{t}}, \bar{x}_{t} \in \bar{\omega}$.

Let $\beta=\left\{\beta_{i}\left(\bar{x}_{\tau}\right)\right\}, i=1, \ldots, n ; \tau=0, \ldots, T ; \bar{x}_{\tau} \in \bar{\omega}$, denote the Payoff Distribution Procedure (PDP) for the cooperative solution $\left(p_{1}, \ldots, p_{n}\right)=\left(p_{1}^{\bar{x}_{0}}, \ldots, p_{n}^{\bar{x}_{0}}\right)$ (see, e.g., $\quad$ Petrosyan and Danilov, 1979; $\quad$ Petrosyan and Kuzyutin, 2000; Yeung and Petrosyan, 2012; Haurie et al., 2012; Kuzyutin et al., 2018), i.e., a time schedule of actual payments to the players along the cooperative path. Namely, $\beta_{i}\left(\bar{x}_{\tau}\right)$ denotes the actual current payment that the $i$-th player receives at node $\bar{x}_{\tau} \in \bar{\omega}$ instead of $h_{i}\left(\bar{x}_{\tau}\right)$ if the players employ payoff distribution procedure $\beta$.

Let us remind several advantageous properties of a PDP $\beta$ (see (Petrosyan and Danilov, 1979; Petrosyan and Kuzyutin, 2000; Kuzyutin et al., 2018; Kuzyutin and Smirnova, 2020) for details). 
Definition 4. (Kuzyutin and Smirnova, 2020) The PDP $\beta$ for the payoff vector $\left(p_{1}, \ldots, p_{n}\right)$ meeting (5) satisfies the subgame efficiency condition, if for all $\bar{x}_{t} \in$ $\bar{\omega}=\left(\bar{x}_{0}, \ldots, \bar{x}_{T}\right)$

$$
\sum_{\tau=t}^{T} \beta_{i}\left(\bar{x}_{\tau}\right)=\widetilde{\beta}_{i}\left(\bar{x}_{t}, \bar{x}_{t+1}, \ldots, \bar{x}_{T}\right)=\widetilde{\beta}_{i}\left(\bar{\omega}^{\bar{x}_{t}}\right)=p_{i}^{\bar{x}_{t}} .
$$

Condition (6) for $t=0$ is usually called the efficiency in the whole game (Petrosyan and Danilov, 1979; Petrosyan and Kuzyutin, 2000; Kuzyutin and Smirnova, 2020).

Definition 5. (Petrosyan and Danilov, 1979; $\quad$ Petrosyan and Kuzyutin, 2000; Kuzyutin et al., 2018) The PDP $\beta=\left\{\beta_{i}\left(\bar{x}_{\tau}\right)\right\}$ satisfies the strict balance condition if for each node $\bar{x}_{\tau} \in \bar{\omega}, \tau=0, \ldots, T$

$$
\sum_{i \in N} \beta_{i}\left(\bar{x}_{\tau}\right)=\sum_{i \in N} h_{i}\left(\bar{x}_{\tau}\right)
$$

Remark 2. The strict balance constraint (7) for a PDP $\beta$ follows from the subgame efficiency condition (6).

In the paper, we assume that PDP $\beta$ should satisfy non-negativity constraint:

$$
\beta_{i}\left(\bar{x}_{\tau}\right) \geqslant 0 \quad \forall i \in N \quad \forall t=0, \ldots, T .
$$

We introduced in (Kuzyutin and Smirnova, 2021) such a PDP $\beta$ that all active coalitions $S \subset N$ have an incentive to follow a cooperative agreement $\left(p_{1}^{\bar{x}_{t}}, \ldots, p_{n}^{\bar{x}_{t}}\right)$ in each subgame $\Gamma^{\bar{x}_{t}}, \bar{x}_{t} \in \bar{\omega}$, along the cooperative history. Let us provide the definition of the $\beta$-subgame-perfect core (Kuzyutin and Smirnova, 2021) below for the sake of completeness.

Suppose that a coalition $S \subset N$ follows a cooperative scenario (namely, a PDP $\beta$ is implemented along the cooperative path from the root $\bar{x}_{0}$ till some intermediate node $\bar{x}_{t} \in \bar{\omega}, 1 \leqslant t \leqslant T-1$ ), but then decides to deviate from the cooperative mode in the subgame $\Gamma^{\bar{x}_{t}}$ (S should be active in $\left.\Gamma^{\bar{x}_{t}}\right)$. Then, assuming that the remaining players form singletons (see (Chander, 2007; Chander and Wooders, 2020) for discussion), the highest payoff a coalition $S$ could reach in the whole game $\Gamma^{x_{0}}$ is equal to $\sum_{\tau=0}^{t-1} \beta_{S}\left(\bar{x}_{\tau}\right)+\gamma\left(S ; \bar{x}_{t}\right)$.

If one suppose that

$$
\sum_{\tau=0}^{t-1} \beta_{S}\left(\bar{x}_{\tau}\right)+\gamma\left(S ; \bar{x}_{t}\right) \leqslant \sum_{\tau=0}^{t-1} \beta_{S}\left(\bar{x}_{\tau}\right)+\sum_{\tau=t}^{T} \beta_{S}\left(\bar{x}_{\tau}\right),
$$

then coalition $S$ has no reason to deviate at $\bar{x}_{t}$ from the cooperative scenario.

If we simplify (9) and take into account $t=0$, we get the following condition for the "subgame sustainability" of a cooperative agreement $\left(S\right.$ is active at $\left.\bar{x}_{t}\right)$ :

$$
\gamma\left(S ; \bar{x}_{t}\right) \leqslant \sum_{\tau=t}^{T} \beta_{S}\left(\bar{x}_{\tau}\right), \quad \bar{x}_{t} \in \bar{\omega}, \quad t=0, \ldots, T-1 .
$$

Note that inequality (10) implies the same sustainability of the cooperative agreement property as inequality (2) in the S-P Core definition. 
Definition 6. (Kuzyutin and Smirnova, 2021). The set of all payoff distribution procedures $\beta$ meeting (6), (5), (7), (8), and (10) is called the $\beta$-subgame-perfect core $\left(\beta-S-P\right.$ Core) for an extensive-form game $\Gamma^{x_{0}} \in G^{P}(n)$.

Let us use again 2-person extensive-form game from Example 1 to demonstrate a proposed extension of the S-P Core based on the payoff distribution procedure approach.

Example 2. (A 2-player extensive-form game with payoffs defined at each node: $\beta$-S-P Core.)

Hereinafter suppose that the players' payoffs in the extensive-form game $\Gamma^{x_{0}}$ from Ex. 1 are determined at all nodes $x \in P$ (this payoffs $\left(h_{1}(x), h_{2}(x)\right)^{T}$ are written in the game tree while the total payoffs collected along each history are written below corresponding terminal nodes).

Constraints (6), (5) for $t=0$ take the form

$$
\begin{gathered}
\beta_{1}\left(x_{0}\right)+\beta_{1}\left(x_{2}\right)+\beta_{1}\left(x_{6}\right)+\beta_{1}\left(z_{7}\right)=\widetilde{\beta}_{1}(\bar{\omega})=p_{1}, \\
\beta_{2}\left(x_{0}\right)+\beta_{2}\left(x_{2}\right)+\beta_{2}\left(x_{6}\right)+\beta_{2}\left(z_{7}\right)=\widetilde{\beta}_{2}(\bar{\omega})=p_{2}, \\
p_{1}+p_{2}=24 .
\end{gathered}
$$

Let us write conditions (10), for instance, in the subgame $\Gamma^{x_{2}}$ along the cooperative history for all coalitions $S$ which are active at $x_{2}$ :

$$
\left\{\begin{array}{l}
\gamma\left(N ; x_{2}\right)=18 \leqslant \beta_{1}\left(x_{2}\right)+\beta_{2}\left(x_{2}\right)+\beta_{1}\left(x_{6}\right)+\beta_{2}\left(x_{6}\right)+\beta_{1}\left(z_{7}\right)+\beta_{2}\left(z_{7}\right), \\
\gamma\left(\{1\} ; x_{2}\right)=10 \leqslant \beta_{1}\left(x_{2}\right)+\beta_{1}\left(x_{6}\right)+\beta_{1}\left(z_{7}\right), \\
\gamma\left(\{2\} ; x_{2}\right)=7 \leqslant \beta_{2}\left(x_{2}\right)+\beta_{2}\left(x_{6}\right)+\beta_{2}\left(z_{7}\right) .
\end{array}\right.
$$

Note that the first constraint in (11) is binding due to strict balance conditions (7).

Straightforward verification shows that the system (5)-(8) and (10) for this extensive-form game is compatible, and therefore the $\beta$-S-P Core is non-empty. Moreover, each PDP $\beta$ from the $\beta$-S-P Core has to satisfy the following condition:

$$
\widetilde{\beta}_{1}=15+\varepsilon, \quad \widetilde{\beta}_{2}=9-\varepsilon, \quad-1 \leqslant \varepsilon \leqslant 1 .
$$

Let us present two examples of the payoff distribution procedures $\beta$ from the $\beta$-S-P Core. The first PDP $\beta$ implies that the players split equally the total benefit from cooperation: $\widetilde{\beta}_{1}=15, \quad \widetilde{\beta}_{2}=9$;

\begin{tabular}{|c|c|c|c|c|}
\hline $\bar{\omega}$ & $x_{0}$ & $x_{2}$ & $x_{6}$ & $z_{7}$ \\
\hline$\beta_{1}\left(x_{t}\right)$ & 4 & 1 & 2 & 8 \\
\hline$\beta_{2}\left(x_{t}\right)$ & 2 & 4 & 2 & 1 \\
\hline
\end{tabular}

Another PDP $\beta^{\prime}$ implies that the second player receives all the benefit from cooperation: $\widetilde{\beta}_{1}^{\prime}=14, \quad \widetilde{\beta}_{2}^{\prime}=10$;

\begin{tabular}{|c|c|c|c|c|}
\hline $\bar{\omega}$ & $x_{0}$ & $x_{2}$ & $x_{6}$ & $z_{7}$ \\
\hline$\beta_{1}^{\prime}\left(x_{t}\right)$ & 3 & 1 & 2 & 8 \\
\hline$\beta_{2}^{\prime}\left(x_{t}\right)$ & 3 & 4 & 2 & 1 \\
\hline
\end{tabular}




\section{A strategic support of the $\beta-S-P$ Core}

If the players redistribute current payoffs at each node along the cooperative path according to some PDP $\beta$ it is interesting to consider a related (non-cooperative) game $\Gamma_{\beta}^{x_{0}}$ which differs from the original game $\Gamma^{x_{0}}$ only in that the payoffs $h_{i}\left(\bar{x}_{t}\right)$, $i \in N$, at every node $\bar{x}_{t} \in \bar{\omega}$ in the cooperative path are replaced by $\beta_{i}\left(\bar{x}_{t}\right)$. This approach was used earlier, in particular, in (Petrosyan and Kuzyutin, 2000) to introduce a "regularized game" for differential and multistage cooperative game and in (Chander and Wooders, 2020) to define a "strategic transform" of a game in an extensive form with terminal payoffs.

As it was proved in (Kuzyutin and Smirnova, 2021), if $\beta$-S-P Core is non-empty then there exists a SPE $\underline{u}$ in a non-cooperative related game $\Gamma_{\beta}^{x_{0}}$ that generates exactly a cooperative path $\bar{\omega}=\omega(\underline{u})$ with a SPE payoffs vector $H_{i}(\underline{u}), \quad i \in N$, from the $\beta$-S-P Core of the original game $\Gamma^{x_{0}}$. This property means that a cooperative scenario based on the PDP $\beta$ from the $\beta$-S-P Core is strategically supported (i.e., could be implemented as a subgame-perfect equilibrium) in a closely related noncooperative game $\Gamma_{\beta}^{x_{0}}$.

Proposition 1. (Kuzyutin and Smirnova, 2021). Let the $\beta$-subgame-perfect core of an extensive-form game $\Gamma^{x_{0}} \in G^{P}(n)$ is non-empty, and $\beta=\beta_{i}\left(\bar{x}_{t}\right), \quad i \in$ $N, \quad \bar{x}_{t} \in \bar{\omega}$, is a payoff distribution procedure from the $\beta-S-P$ Core. Then there exists a subgame-perfect equilibria $\underline{u}=\left(\underline{u}_{1}, \ldots, \underline{u}_{n}\right)$ in a related non-cooperative game $\Gamma_{\beta}^{x_{0}}$ which generates a cooperative history $\bar{\omega}=\left(\bar{x}_{0}, \ldots, \bar{x}_{T}\right)$ with a SPE payoffs vector $H_{i}(\underline{u})=\sum_{t=0}^{T} \beta_{i}\left(\bar{x}_{t}\right)=\widetilde{\beta}_{i}(\bar{\omega}), \quad i \in N$.

A refinement of Nash equilibria concept called Strong NE (SNE) was introduced in (Aumann, 1976) for strategic games. As it turns out, if an extensive game $\Gamma \subset$ $G^{P}(n)$ admits a unique subgame perfect SNE then $\beta$-S-P Core consists of the unique simplest payoff distribution procedure.

Definition 7. (Chander and Wooders, 2020) A pure strategy profile $\underline{u}=\left(\underline{u}_{1}\right.$, $\left.\ldots, \underline{u}_{n}\right)$ is a subgame perfect SNE of $\Gamma^{x_{0}} \subset G^{P}(n)$ if $\underline{u}$ is a SPE of each induced game $\Gamma^{x_{0}, S}, \quad S \subset N$.

If an extensive game possesses subgame perfect SNE $\underline{u}$ then no coalition has a profitable joint deviation from $\underline{u}$ in any subgame.

Note that a pure strategy definition for extensive game originally implies some redundancy (see, for instance, (Kuhn, 1953; Myerson, 1997; Petrosyan and Kuzyutin, 2000) for details), namely two strategy profiles could define different choices of some players at some nodes but generate the same path in the game tree and the same players' payoffs. In what follows, we will refer to "a game $\Gamma$ possesses a unique SPE or $\Gamma$ has multiple SPE but all of them generate the same path in the game tree" as "a game $\Gamma$ admits a unique SPE".

Proposition 2. Let each induced game $\Gamma^{x_{0}, S}, S \subset N$, of the game $\Gamma^{x_{0}} \subset G^{P}(n)$ admits a unique SPE. If $\Gamma^{x_{0}}$ admits a subgame perfect $S N E$ then this subgame perfect $S N E$ is unique and $\beta-S-P$ Core consists of the unique payoff distribution procedure, namely:

$$
\beta_{i}\left(\bar{x}_{t}\right)=h_{i}\left(\bar{x}_{t}\right), \quad \bar{x}_{t} \in \bar{\omega} .
$$


Proof. The proof of the first statement is straightforward and has been already provided in (Chander and Wooders, 2020). Let $\underline{u}=\left(\underline{u}_{1}, \ldots, \underline{u}_{n}\right)$ denote the unique subgame perfect SNE in $\Gamma^{x_{0}}$.

Since $\underline{u}$ is a unique SPE in $\Gamma^{x_{0}, N}$ it generates exactly the cooperative history $\bar{\omega}=\left(\bar{x}_{0}, \ldots, \bar{x}_{T}\right)$ in the game tree. Moreover, a unique SPE at each induced game

$\Gamma^{x_{0}, S}, \quad S \subset N$, is $\underline{u}$ and every induced subgame $\Gamma^{\bar{x}_{t}, S}, \quad S \subset N, \quad t=0, \ldots, T-1$ admits a unique SPE (namely, the restriction of $\underline{u}$ onto the subgame $\Gamma^{\bar{x}_{t}, S}$ ) that generates a SPE history $\bar{\omega}^{\bar{x}_{t}}=\left(\bar{x}_{t}, \ldots, \bar{x}_{T}\right)$.

Then (10) takes the form

$$
\gamma\left(S ; \bar{x}_{t}\right)=\sum_{\tau=t}^{T} h_{S}\left(\bar{x}_{\tau}\right) \leqslant \sum_{\tau=t}^{T} \beta_{S}\left(\bar{x}_{\tau}\right), \quad t=0, \ldots, T-1 .
$$

Taking (5), (6) and the strict balance condition (7) into account we obtain from (13) by backwards induction that

$$
\beta_{i}\left(\bar{x}_{t}\right)=h_{i}\left(\bar{x}_{t}\right), \quad \bar{x}_{t} \in \bar{\omega} .
$$

Hence, there exists a unique PDP $\beta$ in the $\beta$-S-P Core which implies no transfers between the players while the game unfolds along the cooperative history $\bar{\omega}$.

Corollary 1. If a two-player extensive-form game $\Gamma^{x_{0}} \in G^{P}(2)$ admits a unique $S P E$ which is Pareto-efficient, the $\beta-S-P$ Core consists of the unique PDP (12).

It is worth noting that the $\beta$-S-P Core for extensive-form games is a weaker concept than the subgame-perfect SNE. For instance, the extensive game from Example 2 possesses a non-empty $\beta$-S-P Core, but has no subgame-perfect SNE.

\section{Refinement of the $\beta$-S-P Core}

If the $\beta$-S-P Core of an extensive-form game is non-empty in general it consists of multiple payoff distributions procedures.

Proposition 3. (Kuzyutin and Smirnova, 2021). If the $\beta-S-P$ Core of an extensiveform game $\Gamma \in G^{P}(n)$ is non-empty then it is a (closed) convex polytope $B$ in $R^{n \times(T+1)}$.

It is worth noting that the main purpose of each player $j$ when selecting a unique PDP $\beta$ from $\beta$-S-P Core is the value $p_{j}=\widetilde{\beta}_{j}(\bar{\omega})$ which the $j$-th player will get according to the cooperative agreement, given that distribution $\left\{\beta_{i}\left(\bar{x}_{t}\right)\right\}, \quad \bar{x}_{t} \in \bar{\omega}$, of the $\widetilde{\beta}_{i}(\bar{\omega})$ along the cooperative path satisfies additional constraints $(6),(8)$ and (10).

Let us suggest several optimization based approaches for the $\beta$-S-P Core refinement, i.e., some rules for selecting $\widetilde{\beta}_{j}(\bar{\omega}), \quad j \in N$. One approach is to maximize the benefit of some (target) player $j$ from cooperation:

$$
\widetilde{\beta}_{j}-\gamma\left(\{j\} ; x_{0}\right) \rightarrow \max _{\beta \in B}
$$

Note that (14) is a linear programming problem which has a solution due to Prop. 3. If one apply this approach to the game in Ex.2 assuming that the 2-nd player's payoff $\beta_{2}$ is the goal function the resulting PDP will be $\beta^{\prime}: \widetilde{\beta}_{1}^{\prime}=14, \widetilde{\beta}_{2}^{\prime}=10$. 
Another approach is to adopt some bargaining solution (see, e.g. Moulin, 1988; Castañr et al., 2021) to choose a unique vector $\left(\widetilde{\beta}_{j}\right)_{j \in N}$ from $B$ when the subgame perfect equilibrium payoffs vector $\left(\gamma\left(\{j\} ; x_{0}\right)\right)_{j \in N}$ serves as a disagreement point (point of the "status quo"). For instance, when employing the symmetric Nash bargaining solution, the corresponding problem takes the form

$$
\prod_{i \in N}\left(\widetilde{\beta}_{i}-\gamma\left(\{i\} ; x_{0}\right)\right) \rightarrow \max _{\beta \in B} .
$$

If we apply approach (15) to the game in Ex.2 we should choose PDP $\beta: \widetilde{\beta}_{1}=$ $15, \widetilde{\beta}_{2}=9$.

Let us focus in the paper on the third approach (introduced in (Kuzyutin and Smirnova, 2021)) which takes care of the relative benefit from cooperation $(\mathrm{RBC})$ of the least winning player. Let $\Delta_{j}$ denote the absolute range of the $j$-th player's payoffs in $\Gamma^{x_{0}}$. Then $\frac{\widetilde{\beta}_{j}-\gamma\left(\{j\} ; x_{0}\right)}{\Delta_{j}}$ could be interpreted as the relative benefit of player $j$ from cooperation according to the PDP $\beta$. Then, the players are expected to find the solution of the following optimization problem

$$
\max _{\beta \in B} \min _{j \in N} \frac{\widetilde{\beta}_{j}-\gamma\left(\{j\} ; x_{0}\right)}{\Delta_{j}} .
$$

Remark 3. For two-player game problem (16) takes the form

$$
\frac{\widetilde{\beta}_{1}-\gamma\left(\{1\} ; x_{0}\right)}{\Delta_{1}}=\frac{\widetilde{\beta}_{2}-\gamma\left(\{2\} ; x_{0}\right)}{\Delta_{2}} .
$$

Let us illustrate the latter approach using 2-person game in Ex. 2:

$$
\frac{\widetilde{\beta}_{1}-14}{16-6}=\frac{\widetilde{\beta}_{2}-8}{12-6} \Longleftrightarrow \frac{(15+\epsilon)-14}{10}=\frac{(9-\epsilon)-8}{6} \Longleftrightarrow \epsilon=0.2 .
$$

Hence, $\widetilde{\beta}_{1}=15.2, \widetilde{\beta}_{2}=8.8$, while the exact distribution of $\widetilde{\beta}_{j}$ along the cooperative history $\bar{\omega}$ satisfies constraints $(7),(8)$ and (10). For instance, the following PDP $\beta^{\prime \prime}$ meets all the constraints.

\begin{tabular}{|c|c|c|c|c|c|}
\hline $\bar{\omega}$ & $x_{0}$ & $x_{2}$ & $x_{6}$ & $x_{7}$ & $\widetilde{\beta}_{j}^{\prime \prime}(\bar{\omega})$ \\
\hline$\beta_{1}^{\prime \prime}\left(x_{t}\right)$ & 4.2 & 1 & 2 & 8 & 15.2 \\
\hline$\beta_{2}^{\prime \prime}\left(x_{t}\right)$ & 1.8 & 4 & 2 & 1 & 8.8 \\
\hline
\end{tabular}

We will refer later to the latter approach for the $\beta$-S-P Core refinement as the maxmin $R B C$ rule. Note that different approach to the evaluation of the relative benefit from cooperation (as well as for the "value of the preexisting knowledge") was proposed in (Chebotareva et al., 2021) for differential games.

Another way to contract the $\beta$-S-P Core is to assume that a PDP $\beta$ from the Core has to satisfy some additional properties. For instance, let us consider the irrationalbehavior-proof property for cooperative solution that is implemented via IDP $\beta$ in an extensive-form game (this property was firstly introduced in (Yeung, 2006) for cooperative differential games). 
Definition 8. (Yeung, 2006; Kuzyutin and Nikitina, 2017; Kuzyutin et al., 2019b) The PDP $\beta$ for the payoff vector $\left(p_{1}, \ldots, p_{n}\right)$ meeting (5), (6) satisfies irrationalbehavior-proof (IBP) property (for coalitions) if at each node $\bar{x}_{t} \in \bar{\omega}=\left(\bar{x}_{0}, \ldots, \bar{x}_{T}\right)$, $t=1, \ldots, T-1$, for all coalitions $S$ which are active at $\bar{x}_{t}$ the following inequality holds:

$$
\sum_{\tau=0}^{t-1} \beta_{S}\left(\bar{x}_{\tau}\right)+\gamma\left(S ; \bar{x}_{t}\right) \geqslant \gamma\left(S ; x_{0}\right) .
$$

Inequality (18) means that each active coalition $S$ has an incentive to cooperate (at least from the origin $x_{0}$ until the intermediate node $\bar{x}_{t}$ will be reached) even if $S$ assumes that the cooperative scenario could be broken down at $\bar{x}_{t}$ because of the "irrational behavior" of some other players.

Note that formulae (9) and (18) provide estimations of the coalition $S$ total payoff given the following mode of partial cooperation: the players cooperate along the cooperative history from $x_{0}$ till $\bar{x}_{t}$ and then switch to the non-cooperative (namely - SPE) behavior.

Let us check whether two payoff distribution procedures $\beta$ and $\beta^{\prime}$ in Example 2 satisfy IBP property. It turns out that the first PDP $\beta$ satisfies IBP conditions (18) whereas the second PDP $\beta^{\prime}$ does not. For instance, for coalition $S=\{1\}$ inequalities (18) at node $x_{2}$ take the form:

$$
\begin{aligned}
& \beta_{1}\left(x_{0}\right)+\gamma\left(\{1\} ; x_{2}\right)=4+10 \geqslant 14=\gamma\left(\{1\} ; x_{0}\right), \\
& \beta_{1}^{\prime}\left(x_{0}\right)+\gamma\left(\{1\} ; x_{2}\right)=3+10 \geqslant 14=\gamma\left(\{1\} ; x_{0}\right) .
\end{aligned}
$$

Hence, when the $\beta$-S-P Core consists of multiple PDP, one can use the irrationalbehavior-proof condition (18) as a rule for the $\beta$-S-P Core refinement.

\section{7. $\beta$-S-P Core for fishery-management model}

To demonstrate how $\beta$-S-P Core could be implemented in the dynamic models of renewable resource extraction (see, e.g., Levhari and Mirman, 1980; Mazalov and Rettiyeva, 2011; Breton et al., 2019; Chander, 2017; Ougolnitsky and Usov, 2019; Mazalov et al., 2021) we use a 2-player fishery-management model in extensive form introduced in (Kuzyutin and Smirnova, 2021) which is a finite version of the original fishery-management model (Levhari and Mirman, 1980) that has been studied in (Haurie et al., 2012). We suppose in the paper that competing countries equally evaluate the worth of the resource (fish biomass) remainder after the resource extraction (fishery) process ends (namely, $K_{1}=K_{2}=1$ ), whereas in (Kuzyutin and Smirnova, 2021) the players differently appreciate the resource remainder (the reasons for asymmetric environmental valuation were discussed, e.g., in (Cabo and Tidball, 2021)). Note, that cooperative path and payoff distribution procedure from the $\beta$-S-P Core which are designed below for symmetric case es-

sentially differ from the cooperative solution that was obtained in (Kuzyutin and Smirnova, 2021) for the case of asymmetric environmental valuation.

Example 3. (A 2-player symmetric fishery-management model in extensive form).

Let $y(t)$ denote a (normalized) fish amount in year $t, t=0,1, \ldots, T$, that evolves according to the equation

$$
y(t+1)=a \cdot y(t)
$$


where $a>1$ denotes the annual net growth rate. Assume that two players (e. g., companies or countries) exploit the fishery and let $u_{j}(t) \geq 0$ denote the harvest of player $j$ in year $t$. Given the initial condition $y(0)=y^{0}$ the system dynamics is described by the state equation

$$
y(t+1)=a \cdot\left(y(t)-\left(u_{1}(t)+u_{2}(t)\right)\right),
$$

where $0 \leq u_{1}(t)+u_{2}(t) \leq y(t)$.

The objective function of player $j$ has the following form

$$
H_{j}(\cdot)=\sum_{t=0}^{T-1} \delta_{j}^{t} \sqrt{u_{j}(t)}+K_{j} \cdot \delta_{j}^{T} \sqrt{y(T)}, j=1,2,
$$

where $\delta_{j} \in[0,1)$ is the $j$ th player's discount factor while $K_{j}>0$ describes how the $j$ th player values the fish biomass remainder (fishery's scrap after the exploitation period).

Some additional assumptions are accepted in (Kuzyutin and Smirnova, 2021) to embed the fishery-management model (19)-(20) into an extensive-form game framework. Firstly, suppose that both players can fish out at only two levels: Low $\left(u_{j}^{L}=L_{j}\right)$ or High $\left(u_{j}^{H}=H_{j}\right)$ and consider two-year model, i. e., $T=2$. Further, to obtain a game with perfect information we suppose that in every year player 1 moves (i.e. chooses a particular level of $u_{1}$ ) first.

The resulting discrete-time symmetric fishery-management model in extensive form for particular parameter values: $y(0)=10 ; a=1.25 ; u_{j}^{H}=H_{j}=3, u_{j}^{L}=$ $L_{j}=1 ; \delta_{1}=\delta_{2}=\delta=0.9 ; K_{1}=K_{2}=1$, is presented in figure 2. Note that $P_{1}=\left\{x_{0}, x_{3}, x_{4}, x_{5}, x_{6}\right\}, P_{2}=\left\{x_{1}, x_{2}, x_{7}-x_{14}\right\}, P_{n+1}=\left\{z_{1}, \ldots, z_{16}\right\}$, the right alternative at every node $x_{k} \in P_{j}$ corresponds to high level of fishery efforts (harvest) $\left(u_{j}\left(x_{k}\right)=H_{j}\right)$. It is worth noting that the current payoffs $\sqrt{u_{j}(0)}$ in $x_{1}-x_{6}$ correspond to the first period of fishery process (i. e., $\delta^{0}=1$ according to (20)) whereas the current payoffs $\sqrt{u_{j}(1)}$ in $x_{7}-x_{14}$ and $z_{1}-z_{16}$ correspond to the second year (i. e., $\delta^{1}=0.9$ ). The payoffs $h_{j}\left(x_{0}\right), j=1,2$, at the origin $x_{0}$ could be considered as the players' initial assets.

The extensive-form game described above possesses a unique SPE (the corresponding equilibrium choices at every decision node are presented as dotted lines in fig. 2). This SPE generates the path $\omega^{S P E}=\left(x_{0}, x_{2}, x_{5}, x_{12}, z_{12}\right)$ with the resulting non-cooperative payoffs $(5.4 ; 4.67)$.

The cooperative path $\bar{\omega}=\left(x_{0}, x_{1}, x_{3}, x_{7}, z_{1}\right)$, which is emphasized in bold in fig. 2, corresponds to the highest fish biomass remainder (both environmentally concerned players each year fish out at low level) and implies maximal summary payoff $\widetilde{h}_{1}(\bar{\omega})+\widetilde{h}_{2}(\bar{\omega})=5.46+5.46=10.92$.

Note that the system (5)-(8) and (10) for the extensive-form fishery-management game is compatible, thus the $\beta$-S-P Core is non-empty. To choose a payment vector $\left(\widetilde{\beta}_{1}(\bar{\omega}), \widetilde{\beta}_{2}(\bar{\omega})\right)$ from the $\beta$-S-P Core we employ the maxmin RBC rule. Using the notations $\widetilde{\beta}_{1}(\bar{\omega})=5.46+\varepsilon, \widetilde{\beta}_{2}(\bar{\omega})=5.46-\varepsilon$, we obtain equation (17) in the form

$$
\frac{(5.46+\varepsilon)-5.4}{5.98-4.54}=\frac{(5.46-\varepsilon)-4.67}{5.98-4.01} \Longleftrightarrow \varepsilon=0.3 \text {. }
$$

Hence, $\widetilde{\beta}_{1}(\bar{\omega})=5.76, \widetilde{\beta}_{2}(\bar{\omega})=5.16$. The table below presents an example of particular PDP $\beta$ from the $\beta$-S-P Core that was determined via backwards induction and 


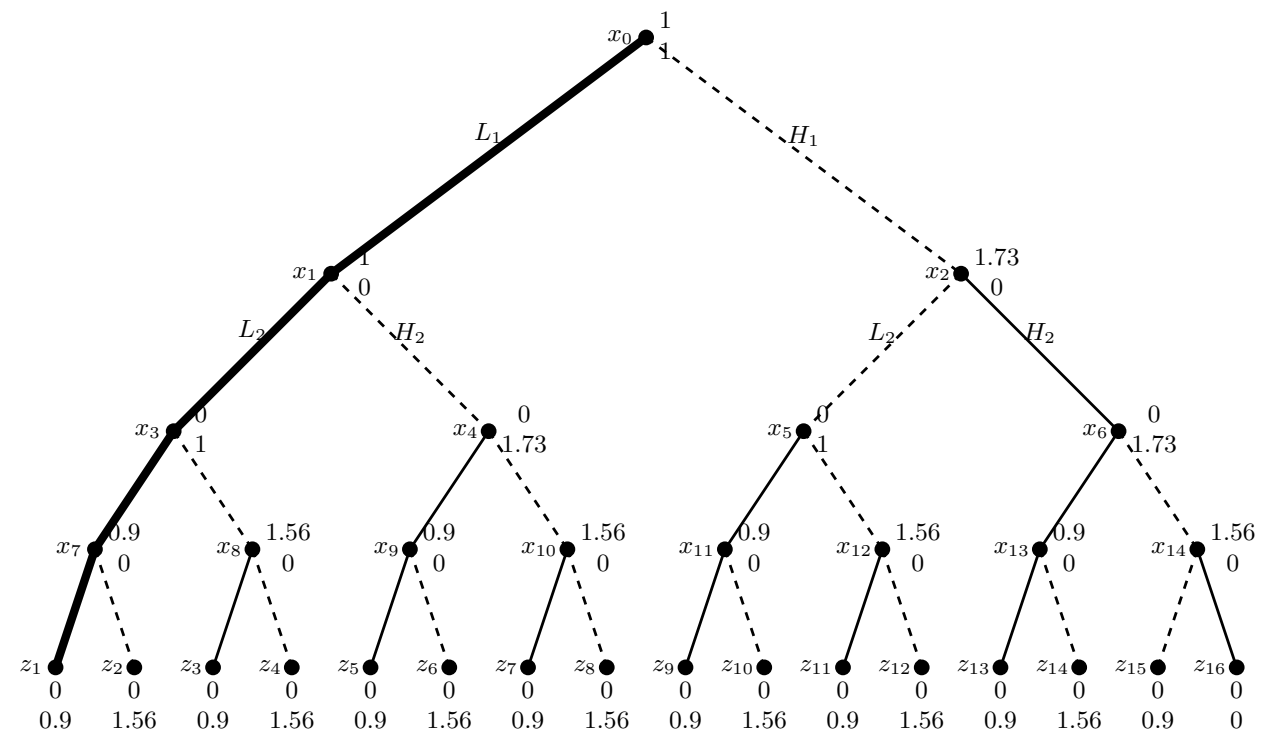

Value of the fish biomass remainder $\beta^{2} \sqrt{y(2)}$ :

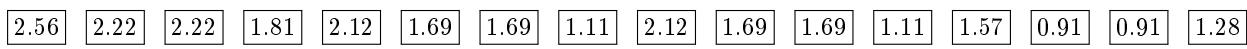

Total players' payoffs (20) :

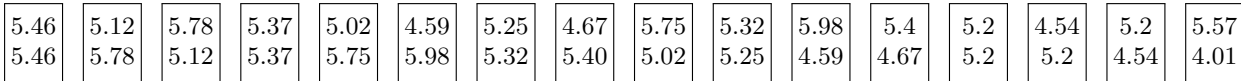

Fig. 2. Fishery-management symmetric 2-player model in extensive form

implies no payoff transfers at terminal node $z_{1}$ and minimal transfers between the players at decision nodes in the cooperative path.

\begin{tabular}{|c||c|c|c|c|c||c|}
\hline $\bar{\omega}$ & $x_{0}$ & $x_{1}$ & $x_{3}$ & $x_{7}$ & $z_{1}$ & $\widetilde{\beta}_{j}(\bar{\omega})$ \\
\hline$\beta_{1}\left(x_{t}\right)$ & 1.39 & 1 & 0.23 & 0.58 & 2.56 & 5.76 \\
\hline$\beta_{2}\left(x_{t}\right)$ & 0.61 & 0 & 0.77 & 0.32 & 3.46 & 5.16 \\
\hline$\beta_{1}\left(x_{t}\right)-h_{1}\left(x_{t}\right)$ & 0.39 & 0 & 0.23 & -0.32 & 0 & 0.3 \\
\hline
\end{tabular}

Note that the exact values of the payoff transfers (from player 2 to player 1 ) which are necessary at each node of the cooperative path to ensure the sustainability of the cooperative agreement are given in the lowest table row.

\section{Conclusion}

The paper contributes to the theory of cooperative behavior in extensive-form games. Firstly, we compare the subgame-perfect core (Chander and Wooders, 2020) and $\beta$-S-P Core (Kuzyutin and Smirnova, 2021) and demonstrate in Sections 3 and 4 that the latter solution is more powerful tool to implement a cooperative agreement (this implication was briefly noted in (Kuzyutin and Smirnova, 2021) without discussion). Secondarily, in Section 5 we reveal relationship between strong Nash equilibria and $\beta$-S-P Core for the class of extensive-form games with perfect information and payoffs defined at each node. Lastly, in Section 6 we propose several 
approaches for the $\beta$-S-P Core refinement (some of them require an optimization problem solution, others imply that particular PDP from the $\beta$-S-P Core has to satisfy additional properties like irrational-behavior-proof condition) and illustrate these approaches using two examples.

Note that $\beta$-S-P Core approach is very promising and could be applied to other classes of extensive-form games (e.g., extensive games with chance moves (Kuzyutin and Smirnova, 2020) or extensive-form multicriteria games (Kuzyutin and Nikitina, 2017; Kuzyutin et al., 2018; Kuzyutin et al., 2019b). It is also of interest how one can adapt the $\beta$-S-P Core concept in the discrete-time models of dynamic interaction between several religious and political movements (see, e.g., Tantlevskij et al., 2021; Kuzyutin et al., 2020).

Acknowlegments. The research of the first author was funded by RFBR, project number 21-011-44058.

\section{References}

Aumann, R. (1959). Acceptable points in general cooperative n-person games. In: Contributions to the Theory of Games IV. Princeton University Press: Princeton, N.J.

Breton, M., I. Dahmouni and G. Zaccour (2019). Equilibria in a two-species fishery. Math. Biosci., 309, 78-91.

Cabo, F. and M. Tidball (2021). Cooperation in a Dynamic Setting with Asymmetric Environmental Valuation and Responsibility. Dyn Games Appl., forthcoming. https://doi.org/10.1007/s13235-021-00395-y

Castañer, A., J. Marín-Solano and C. Ribas (2021). A time consistent dynamic bargaining procedure in differential games with heterogeneous discounting. Math. Meth. Oper. Res., forthcoming.

Chander, P. (2007). The gamma-core and coalition formation. Int. J. Game Theory, 35, 539-556. https ://doi .org/10.1007/s00182-006-0067-9

Chander, P. (2017). Subgame-perfect cooperative agreements in a dynamic game of climate change. J. Environ. Econ. Manag., 84(C), 173-188.

Chander, P. and H. Tulkens (1997). The core of an economy with multilateral environmental externalities. Int. J. Game Theory, 26, 379-401.

Chander, P. and M. Wooders (2020). Subgame-perfect cooperation in an extensive game. Journal of Economic Theory, 187, 105017.

Chebotareva, A., Su, S., S. Tretyakova and E. Gromova (2021). On the Value of the Preexisting Knowledge in an Optimal Control of Pollution Emissions. In: Contributions to Game Theory and Management (Petrosyan, L. A. and N. A. Zenkevich, eds), Vol. 14, forthcoming. St. Petersburg State Univ. Press: St. Petersburg.

Crettez, B., N. Hayek and G. Zaccour (2020). Do charities spend more on their social programs when they cooperate than when they compete? Eur. J. Oper. Res., 283, 10551063.

Haurie, A., J. B. Krawczyk and G. Zaccour. (2012). Games and Dynamic Games. Scientific World: Singapore.

Hillas, J. and D. Kvasov (2020). Backward induction in games without perfect recall. Games and Economic Behavior, 124, 207-218. https://doi.org/10.1016/j.geb.2020.08.011

Kuhn, H. (1953). Extensive games and the problem of information. Ann. Math., 28, 193216.

Kuzyutin, D., E. Gromova and Ya. Pankratova (2018). Sustainable cooperation in multicriteria multistage games. Oper. Res. Lett., 46, 557-562.

Kuzyutin, D., Lipko, I., Y. Pankratova and I. Tantlevskij (2020). Cooperation Enforcing in Multistage Multicriteria Game: New Algorithm and Its Implementation. In: Frontiers of 
Dynamic Games (Petrosyan, L., V. Mazalov and N. Zenkevich, eds), Static \& Dynamic Game Theory: Foundations \& Applications. Birkhएouser, Cham.

Kuzyutin, D. and M. Nikitina (2017). An irrational behavior proof condition for multistage multicriteria games. In: Constructive Nonsmooth Analysis and Related Topics (Dedic. to the Memory of V.F.Demyanov), 178-181. IEEE: New York, NY, USA.

Kuzyutin, D., Y. Pankratova and R. Svetlov (2019a). A-Subgame Concept and the Solutions Properties for Multistage Games with Vector Payoffs. In: Frontiers of Dynamic Games (Petrosyan, L., V. Mazalov and N. Zenkevich, eds), Static \& Dynamic Game Theory: Foundations \& Applications. Birkh ouser, Cham.

Kuzyutin, D. and I. Romanenko (1998). On properties of equilibrium solutions for $n$ person games in extensive form. Vestnik St. Petersburg Univ. Math., 3(15), 17-27 (in Russian).

Kuzyutin, D. and N. Smirnova (2020). Subgame Consistent Cooperative Behavior in an Extensive form Game with Chance Moves. Mathematics, 8(7), 1061. https://doi.org/10.3390/math8071061

Kuzyutin, D., Smirnova, N. and Tantlevskij, I. (2021). Subgame-perfect core based on the payoff distribution procedure. Submitted to Economics Letters.

Kuzyutin, D., N. Smirnova and E. Gromova (2019b). Long-term implementation of the cooperative solution in multistage multicriteria game. Oper. Res. Perspect., 6, 100107.

Levhari, D. and L. J. Mirman (1980). The Great Fish War: An Example Using a Dynamic Cournot-Nash solution. The Bell Journal of Economics, 11 (1), 322-334.

Mazalov, V., E. Parilina and J. Zhou (2021). Altruistic-Like Equilibrium in a Differential Game of Renewable Resource Extraction. In: Mathematical Optimization Theory and Operations Research (Pardalos, P., M. Khachay and A. Kazakov, eds). MOTOR 2021. Lecture Notes in Computer Science, 12755. Springer, Cham.

Mazalov, V.V. and A. N. Rettiyeva (2011). The discrete-time bioresource sharing model. J. Appl. Math. Mech., 75, 180-188.

Myerson, R. (1997). Game Theory. Analysis of Conflict. Harvard University Press: Cambridge, MA,USA.

Moulin, H. (1988). Axioms of cooperative decision making. Cambridge University Press: Cambridge, MA, USA.

Nash, J. F. (1950). Equilibrium points in n-person games. Proc. Nat. Acad. Sci. USA, 36, $48-49$.

Ougolnitsky, G. and A. Usov (2019). Spatially distributed differential game theoretic model of fisheries. Mathematics, 7, 732. doi:10.3390/math7080732.

Petrosyan, L. (1977). Time-consistency of solutions in multi-player differential games. Vestnik of Leningrad State University. Series 1. Mathematics. Mechanics. Astronomy, $4,46-52$.

Petrosyan, L. A. and N. N. Danilov (1979). Stability of solutions in non-zero sum differential games with transferable payoffs. Vestnik of Leningrad State University. Series 1. Mathematics. Mechanics. Astronomy, 1, 52-59.

Petrosyan, L. and D. Kuzyutin (2000). Games in Extensive Form: Optimality and Stability. Saint Petersburg University Press: St. Petersburg, Russia (in Russian).

Selten, R. (1975). Reexamination of the Perfectness Concept for Equilibrium Points in Extensive Games. Int. J. Game Theory, 4, 25-55.

Tantlevskij, I., E. Gromova and D. Gromov (2021). Network Analysis of the Interaction between Different Religious and Philosophical Movements in Early Judaism. Philosophies, 6(1), 2. https://www. mdpi.com/2409-9287/6/1/2

Yeung, D. (2006). An irrational-behavior-proof condition in cooperative differential games. International Game Theory Review, 8(4), 739-744.

Yeung, D. and L. Petrosyan (2012). Subgame Consistent Economic Optimization: An Advanced Cooperative Dynamic Game Analysis. Springer: New York, NY, USA. 